\title{
Caracterización Antropométrica y Composición Corporal de Tenistas de Elite Varones y Damas de Chile
}

\author{
Anthropometric Characterization and Body Composition \\ of Male and Female Elite Tennis Players in Chile
}

\author{
Pablo Luna Villouta ${ }^{1,2}$; Luis Felipe Castelli Correia de Campos $^{3}$; Marcelo Paredes Arias ${ }^{4}$; \\ Rodrigo Vargas Vitoria ${ }^{2}$; Cristian Martínez Salazar ${ }^{5} \&$ Nelson Araneda Garces ${ }^{6}$
}

\begin{abstract}
VIllouTA, P. L.; CORREIA DE CAMPOS, L. F. C.; PAREDES, A. M.; VARGAS, V. R.; MARTÍNEZ, S, C. \& ARANEDA, G. N. Caracterización antropométrica y composición corporal de tenistas de elite varones y damas de Chile.Int. J. Morphol., 39(1):84$89,2021$.
\end{abstract}

RESUMEN: El objetivo del estudio fue determinar las características antropométricas y de composición corporal en jóvenes tenistas de elite chilenos. El estudio fue de carácter descriptivo de corte transversal. La muestra de 84 tenistas (58 varones 15,5 \pm 0,76 años y 26 damas, 15,3 $\pm 0,8$ años), fue seleccionada de forma no probabilística por conveniencia. Se evaluaron variables antropométricas de peso, estatura, estatura sentada, pliegues cutáneos (bíceps, tríceps, subescapular, supraespinal, abdominal, muslo anterior y pierna medial), perímetros (brazo flexionado, muslo medio y pierna) y diámetros (biepicondíleo de húmero y del fémur). Se realizaron análisis antropométricos y cálculos para determinar la composición corporal y el somatotipo de los sujetos participantes. Los resultados para los

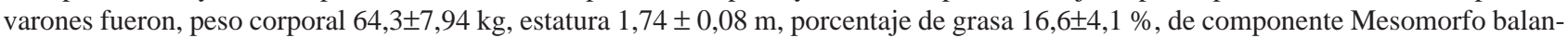

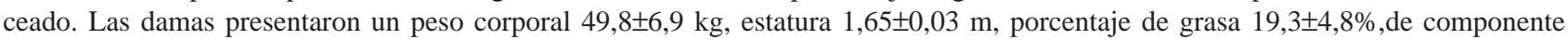
Ectomorfo balanceado. En general, ambos grupos muestran características antropométricas y de composición corporal similares a otros grupos de tenistas juveniles, a excepción del somatotipo en el caso de las damas.

PALABRAS CLAVE: Tenis; Antropometría; Composición corporal; Somatotipo.

\section{INTRODUCCIÓN}

La antropometría es una técnica que utiliza procedimientos simples, no invasivos, económicos y presentando una relativa facilidad en la interpretación de sus resultados (Zemel et al., 1997; Guedes \& Rechenchosky, 2008), siendo consideradas evaluaciones que permiten expresar cuantitativamente la forma del cuerpo.

Las variables antropométricas presentan diversas aplicaciones, ya que permiten caracterizar a los distintos grupos humanos, evaluar el estado nutricional, monitorear el crecimiento físico y verificar cambios en el somatotipo, la proporcionalidad y la composición corporal en diversas fases del desarrollo humano (Cossio-Bolaños et al., 2015). Por lo anterior, la aplicabilidad de la valoración antropométrica es muy amplia, permitiendo el monitoreo tanto de grandes grupos poblaciones como de diversas modalidades deportivas tanto adultas como adolescentes (Pradas de la Fuente et al., 2007).

De esta forma, elinterés por las características antropométricas, la composición corporal y el somatotipo de los diferentes deportes de competición ha aumentado en las últimas décadas (Sanchez et al., 2007), más aún al señalarse que las características morfológicas son importantes para triunfar en un deporte (Malina et al., 2004).

Por lo anterior, el análisis de las características corporales y la composición corporal, constituyen herramientas valiosas, tanto para la selección precoz de la modalidad deportiva más adecuada para un sujeto de acuerdo con

\footnotetext{
${ }^{1}$ Facultad de Educación, Universidad San Sebastián, Concepción, Chile.

${ }^{2}$ Facultad de Ciencias de la Educación, Universidad Católica del Maule, Doctorado en Ciencias de la Actividad Física UCM, Chile.

${ }^{3}$ Facultad de Educación y Humanidades, Universidad del Bío-Bío, Chillán, Chile.

${ }^{4}$ Facultad de Educación, Universidad San Sebastián, Concepción, Chile.

${ }^{5}$ Departamento de Educación física Deporte y Recreación, Universidad de La Frontera, Temuco, Chile.

${ }^{6}$ Departamento de Educación,Universidad de La Frontera, Temuco, Chile.
} 
sus cualidades anatómicas, como para el control de los programas de entrenamiento y competición (Pradas de la Fuente et al.).

Recientemente, se está produciendo un creciente interés en los representantes del tenis juvenil por conocer los factores que influyen en su rendimiento físico, pero solo algunos datos ya han sido publicados (Ziemann et al., 2011).

El tema aún está en desarrollo, ya que poco se conoce acerca de la evolución de las variables antropométricas y del rendimiento físico, aunque parezca particularmente esencial para un entrenamiento efectivo (Ziemann et al.), de manera especial, en la adolescencia que es un período de cambios rápidos y significativos en el organismo, por lo que la evaluación de distintos parámetros asociados al rendimiento deportivo pueden proporcionar a losentrenadores y jugadores una información importante para la toma decisiones y planificación del entrenamiento (Ziemann et al.) como también puede ser esencial para la adecuada selección del talento deportivo (Peña et al., 2018).

Hasta la fecha, la valoración antropométrica y de composición corporal en tenistas, cuenta con algunas publicaciones a nivel internacional (Torres Luque et al., 2006; Sánchez-Muñoz et al., 2007; Ziemann et al.); en específico, a nivel sudamericano, existen muy pocas indagaciones, más aún a nivel juvenil, las que han recogido información de jóvenes tenistas argentinos (Lentini et al., 2004), y brasileños (Pereira, 2001; Cócaro et al., 2012).En este sentido, la evidencia científica señala que es importante abordar de manera más satisfactoria el potencial atlético de los jóvenes tenistas, teniendo como referencia, además, sus niveles de maduración (Kramer et al., 2016).

Por lo tanto, a nuestro entender estos aspectos deberían ser estudiados desde varios puntos de vista, cuyo propósito fundamental radicaría en proporcionar información respecto a las distintas variables asociadas rendimiento deportivo.

A partir de los antecedentes mencionados, se plantea el objetivo de determinar las características antropométricas y de composición corporal en jóvenes tenistas chilenos de entre 14 a 16 años.

\section{MATERIAL Y MÉTODO}

Estudio Descriptivo de corte transversal, donde participaron voluntariamente 84 tenistas juveniles( 58 varones $15,5 \pm 0,76$ años y26 damas, $15,3 \pm 0,8$ años), pertenecien- tes a escuelas de tenis de la Región Metropolitanade Chile. Las instituciones deportivas fueron seleccionadas por su disponibilidad de participación, además se ponderó que contaran con actividades deportivas planificadas y ejecutadas sistemáticamente (al menos cinco veces por semana, con un mínimo de entrenamiento de 12 horas semanales), con participación permanente en torneos regionales, nacionales y/o internacionales, durante al menos el último año, y que además estuvieran a cargo de personal técnico certificado por la Federación de Tenis de Chile (FETECH) o la Federación Internacional de Tenis (ITF).

Como procedimiento, se les envió una carta a los directivos de las escuelas de tenis, declarando el objetivo de la investigación e invitándolos a participar. Luego de esto, se envió un consentimiento informado a los apoderados de los sujetos, para informar respecto del objetivo del estudio, el carácter anónimo y voluntario de la participación, y de las características de las evaluaciones; el consentimiento debía ser firmado autorizando la participación de su pupilo, luego se firmó el asentimiento por parte de cada sujeto, ratificando su participación en las evaluaciones. El estudio contó con la aprobación del comité de ética de la Universidad San Sebastián, Chile (Res. № 512018-20).

Las evaluaciones antropométricas fueron realizadas antes de efectuar cualquier tipo de actividad física en el período de la mañana, en una sala especialmente acondicionada, la que permitió realizar evaluaciones individuales y privadas. Las que estuvieron a cargo de evaluadores capacitados siguiendo los procedimientos estándar de Ross \& Marfell-Jones (1991).

La masa corporal $(\mathrm{kg})$ se midió utilizando una balanza mecánica (Seca 700), con una precisión de 50 grs, que oscila entre 0 a $220 \mathrm{~kg}$, la talla (m), según el plano de Frankfurt sin zapatos, utilizando un estadiómetro de aluminio de marca Seca 220, graduado en milímetros, su escala fue de $(0,60-2,20 \mathrm{~m})$. La altura sentada (altura troncocefálica) fue tomada usando un banco de madera con una altura de $0,50 \mathrm{~m}$, con una escala de medición de 0 a $1,50 \mathrm{~m}$ y con una precisión de $1 \mathrm{~mm}$. En el caso de los pliegues cutáneos, se hizo con una pinza antropométrica HarpendenSkinfold ${ }^{\circledR}$. Los diámetros óseos, con Antropómetro corto Faga ${ }^{\circledR}$, por su parte los perímetros con la cinta antropométrica Lufkin ${ }^{\circledR}$ Metálica.

A partir de las medidas antropométricas, se calcularon diferentes índices y porcentajes con el fin de determinar las características corporales de los tenistas evaluados. Así, el análisis del somatotipo se realizó mediante el método de Carter \& Heath (1990), obteniéndose el valor de los 
tres componentes: endomórfico, mesomórfico y ectomórfico. Por su parte, se calculó del índice de masa corporal (IMC, expresado en $\mathrm{kg} / \mathrm{m}^{2}$ )de acuerdo a los criterios establecidos por la Organización Mundial de la salud (2000). El porcentaje de grasa corporal se calculó con ecuaciones de regresión propuestas por Slaughter et al. (1988). La maduración biológica se determinó por medio de los años de pico de velocidad de crecimiento (PVC), y se predijo por medio de Ecuaciones de regresión tanto para hombres como para mujeres, propuestas por Mirwald et al. (2002).

Los datos obtenidos se analizaron utilizando el Software GraphPadPrism 7.0 y son presentados por medio de la estadística descriptiva de media, desviación estándar, mediana, primer y tercer cuartil, Intervalo de Confianza $95 \%$ y el coeficiente de variación. Para el análisis de la normalidad de la muestra, fue utilizado el test de Shapiro-Wilk para las damas y el Lilliefors Test para los varones. Para comparar las variables antropométricas y de composición corporal entre varones y damas fue utilizado el test t no pareado para las variables paramétricas y la prueba de Mann-Whitney para variables no paramétricas. El nivel de significación utilizado para todas las variables del estudio fue de $p<0,05$.

\section{RESULTADOS}

En las Tablas I y II son presentados los valores de promedio, desviación estándar, mínimo, máximo, primero y tercero cuartiles, el intervalo de confianza $95 \%$ y el coeficiente de varianza de los varones y de las damas, respectivamente. (Tabla I).

En relación a las variables antropométricas se observó diferencia significativa entre los varones y damas para las medidas de Estatura $(\mathrm{V}=1,74 \pm 0,08 \mathrm{~m}, \mathrm{D}=1,65 \pm 0,03$, $\mathrm{p}<0,0001), \mathrm{PC}(\mathrm{V}=64,3 \pm 7,94 \mathrm{~kg}, \mathrm{D}=49,8 \pm 6,9 \mathrm{~kg}$, $\mathrm{p}<0,0001), \mathrm{LB}(\mathrm{V}=27,0 \pm 1,26 \mathrm{~cm}, \mathrm{D}=25,5 \pm 0,4 \mathrm{~cm}$, $\mathrm{p}<0,0001), P M(V=46,6 \pm 4,3 \mathrm{~cm}, \mathrm{D}=43,5 \pm 4,5 \mathrm{~cm}$, $\mathrm{p}<0,003), P G(V=34,8 \pm 4,3 \mathrm{~cm}, \mathrm{D}=31,8 \pm 3,2 \mathrm{~cm}, 0,0002)$ y $\mathrm{PBC}(\mathrm{V}=28,7 \pm 3,0 \mathrm{~cm}, \mathrm{D}=25,7 \pm 4,6 \mathrm{~cm}, \mathrm{p}<0,0007)$ y PVC $(V=0,4 \pm 0,99 \mathrm{~cm}, \mathrm{D}=4,1 \pm 0,9 \mathrm{~cm}, \mathrm{p}<0,001$ ) (Tabla II).

Cuando son comparadas las variables de composición corporal entre ambos sexos, se observó que los varones presentaban valores más altos que las damas en las variables de IMC $\left(\mathrm{V}=21,1 \pm 1,85 \mathrm{~kg} / \mathrm{m}^{2} ; \mathrm{D}=18,3 \pm 2,5 \mathrm{~kg} /\right.$ $\left.\mathrm{m}^{2}, \mathrm{p}<0.001\right)$, Mesomorfia $(\mathrm{V}=3,9 \pm 1,59, \mathrm{D}=3,0 \pm 1,70$, $\mathrm{p}<0,0280)$ y $\mathrm{MLG}(\mathrm{V}=53,6 \pm 6,61 \mathrm{~kg}, \mathrm{D}=40,1 \pm 5,0$, $\mathrm{p}<0,0001)$ y valores inferiores para las variables de Ectomorfia $(\mathrm{V}=3,3 \pm 1,07, \mathrm{D}=4,4 \pm 1,5, \mathrm{p}<0.005)$ y $\%$ $\mathrm{GC}(\mathrm{V}=16,6 \pm 4,10 \%, \mathrm{D}=19,3 \pm 4,8 \%, \mathrm{p}<0,0109)$. Los resultados pueden ser observados en la Figura 1.

Tabla I. Caracterización de las medidas antropométricas y de Composición Corporal de los atletas tenistas varones de 14-16 años.

\begin{tabular}{|c|c|c|c|c|c|c|c|c|c|c|}
\hline \multirow{2}{*}{ Variables } & \multirow{2}{*}{ Media } & \multirow[t]{2}{*}{$\mathrm{DE}$} & \multirow{2}{*}{ Min } & \multirow{2}{*}{$\mathrm{C} 25$} & \multirow{2}{*}{ Mediana } & \multirow{2}{*}{ C75 } & \multirow{2}{*}{ Máx } & \multicolumn{2}{|c|}{ IC $95 \%$} & \multirow[t]{2}{*}{$\mathrm{CV}(\%)$} \\
\hline & & & & & & & & LI & $\mathrm{LS}$ & \\
\hline Edad (años) & 15,5 & 0,76 & 14,0 & 14,9 & 15,5 & 16,1 & 16,6 & 15,1 & 15,9 & 4,9 \\
\hline Estatura (m) & 1,74 & 0,08 & 1,53 & 1,71 & 1,75 & 1,8 & 1,96 & 1,7 & 1,8 & 4,4 \\
\hline $\mathrm{PC}(\mathrm{kg})$ & 64,3 & 7,94 & 42,7 & 60,3 & 65,0 & 69,8 & 80,7 & 63,5 & 67,8 & 12,4 \\
\hline $\operatorname{IMC}\left(\mathrm{kg} / \mathrm{m}^{2}\right)$ & 21,1 & 1,85 & 16,3 & 20,4 & 21,3 & 22,0 & 26,3 & 20,9 & 21,7 & 8,7 \\
\hline $\mathrm{PM}(\mathrm{cm})$ & 46,6 & 4,30 & 33,4 & 44,4 & 46,4 & 49,0 & 60,4 & 46,0 & 47,0 & 9,2 \\
\hline PG (cm) & 34,8 & 4,31 & 23,0 & 32,1 & 35,3 & 37,3 & 51,2 & 33,1 & 37,0 & 12,4 \\
\hline $\mathrm{PBC}(\mathrm{cm})$ & 28,7 & 3,03 & 21,9 & 26,6 & 29,0 & 30,1 & 38,2 & 27,6 & 29,5 & 10,6 \\
\hline $\mathrm{DH}(\mathrm{cm})$ & 6,9 & 0,66 & 5,7 & 6,5 & 6,9 & 7,2 & 9,1 & 6,8 & 7,0 & 9,6 \\
\hline $\mathrm{DF}(\mathrm{cm})$ & 9,3 & 0,72 & 7,6 & 8,8 & 9,2 & 9,9 & 10,9 & 8,9 & 9,7 & 7,7 \\
\hline $\mathrm{LB}(\mathrm{cm})$ & 27,0 & 1,26 & 24,0 & 26,5 & 27,4 & 27,8 & 29,9 & 26,9 & 27,6 & 4,7 \\
\hline$\sum 7 \mathrm{PC}(\mathrm{mm})$ & 81,0 & 21,96 & 48,2 & 66,9 & 74,8 & 90,2 & 177,4 & 71,7 & 84,7 & 27,1 \\
\hline PVC (años) & 0,4 & 0,99 & $-2,1$ & $-0,4$ & 0,6 & 1,2 & 2,2 & 0,2 & 0,9 & 238,7 \\
\hline ENDO & 3,3 & 1,06 & 1,5 & 2,7 & 3,1 & 4,1 & 7,1 & 2,8 & 3,6 & 31,6 \\
\hline MESO & 3,9 & 1,59 & 0,9 & 2,8 & 3,6 & 4,7 & 8,1 & 3,0 & 4,3 & 40,9 \\
\hline ЕСТO & 3,3 & 1,07 & 1,0 & 2,8 & 3,1 & 3,8 & 7,4 & 3,0 & 3,3 & 32,1 \\
\hline MG (kg) & 10,7 & 3,39 & 6,4 & 8,5 & 10,0 & 12,0 & 23,0 & 9,0 & 10,7 & 31,6 \\
\hline MLG (kg) & 53,6 & 6,61 & 34,1 & 50,9 & 54,3 & 58,7 & 64,6 & 53,0 & 57,0 & 12,3 \\
\hline $\mathrm{GC}(\%)$ & 16,6 & 4,10 & 10,0 & 13,5 & 15,6 & 18,4 & 29,6 & 15,0 & 17,2 & 24,7 \\
\hline
\end{tabular}

Nota: PC - Peso Corporal; PM - perímetro Muslo; PG - perímetro gemelo; PBC - Perímetro Brazo Contraído; DH - Diámetro Humero; DF - Diámetro Fémur; LB - longitud del brazo; $\sum 7 \mathrm{PC}$ - sumatoria de los pliegues; PVC - Pico de la velocidad de crecimiento; MG - Masa Grasa; MLG - Masa Libre de Grasa; GC - porcentaje de grasa corporal. 
VILloUTA, P. L.; CORREIA DE CAMPOS, L. F. C.; PAREDES, A. M.; VARGAS, V. R.; MARTínEZ, S, C. \& ARANEDA, G. N. Caracterización antropométrica y composición corporal de tenistas de elite varones y damas de Chile. Int. J. Morphol., 39(1):84-89, 2021.

Tabla II. Caracterización de las medidas antropométricas y de Composición Corporal de los atletas tenistas damas de 14-16 años.

\begin{tabular}{|c|c|c|c|c|c|c|c|c|c|c|}
\hline \multirow{2}{*}{ Variables } & \multirow{2}{*}{ Media } & \multirow{2}{*}{ DE } & \multirow{2}{*}{ Min } & \multirow{2}{*}{$\mathrm{C} 25$} & \multirow{2}{*}{ Mediana } & \multirow{2}{*}{$\mathrm{C} 75$} & \multirow{2}{*}{ Máx } & \multicolumn{2}{|c|}{ IC95\% } & \multirow[t]{2}{*}{$\mathrm{CV}(\%)$} \\
\hline & & & & & & & & LI & $\mathbf{L S}$ & \\
\hline Edad (años) & 15,3 & 0,8 & 14,0 & 14,8 & 15,2 & 15,9 & 16,7 & 14,8 & 15,8 & 5,2 \\
\hline Estatura (m) & 1,65 & 0,03 & 1,57 & 1,64 & 1,65 & 1,68 & 1,69 & 1,64 & 1,67 & 2,1 \\
\hline PC (kg) & 49,8 & 6,9 & 40,5 & 44,2 & 49,3 & 55,8 & 67,0 & 45,6 & 50,6 & 13,8 \\
\hline IMC $\left(\mathrm{kg} / \mathrm{m}^{2}\right)$ & 18,3 & 2,5 & 15,2 & 16,4 & 17,6 & 20,5 & 24,3 & 16,6 & 19,5 & 13,6 \\
\hline $\mathrm{PM}(\mathrm{cm})$ & 43,5 & 4,5 & 31,6 & 40,4 & 44,2 & 46,8 & 49,2 & 41,7 & 46,3 & 10,2 \\
\hline PG (cm) & 31,8 & 3,2 & 22,3 & 30,7 & 32,3 & 33,5 & 37,0 & 31,0 & 33,0 & 10,0 \\
\hline $\mathrm{PBC}(\mathrm{cm})$ & 25,7 & 4,6 & 20,1 & 23,4 & 24,6 & 26,8 & 45,3 & 23,6 & 25,8 & 17,9 \\
\hline $\mathrm{DH}(\mathrm{cm})$ & 6,3 & 0,8 & 4,6 & 6,0 & 6,3 & 6,7 & 8,9 & 6,1 & 6,5 & 11,9 \\
\hline $\mathrm{DF}(\mathrm{cm})$ & 8,6 & 0,6 & 7,1 & 8,2 & 8,5 & 9,2 & 9,6 & 8,2 & 8,9 & 7,1 \\
\hline $\mathrm{LB}(\mathrm{cm})$ & 25,5 & 0,4 & 24,7 & 25,3 & 25,4 & 25,9 & 26,4 & 25,3 & 25,8 & 1,7 \\
\hline$\sum 7 \mathrm{PC}(\mathrm{mm})$ & 80,8 & 29,0 & 51,5 & 56,4 & 77,9 & 90,7 & 165,3 & 58,0 & 85,1 & 35,9 \\
\hline PVC (años) & 4,1 & 0,9 & 2,6 & 3,4 & 4,1 & 4,8 & 5,9 & 3,7 & 4,7 & 22,1 \\
\hline ENDO & 3,2 & 1,3 & 1,6 & 2,6 & 3,0 & 3,7 & 6,5 & 2,6 & 3,5 & 39,4 \\
\hline MESO & 3,0 & 1,7 & $-0,1$ & 2,1 & 2,7 & 3,3 & 9,7 & 2,2 & 3,2 & 57,5 \\
\hline ЕСТО & 4,4 & 1,5 & 1,3 & 3,2 & 4,8 & 5,6 & 6,2 & 3,4 & 5,5 & 33,5 \\
\hline MG (kg) & 9,7 & 3,4 & 6,0 & 6,8 & 9,5 & 11,3 & 20,3 & 7,2 & 10,9 & 34,8 \\
\hline MLG (kg) & 40,1 & 5,0 & 31,3 & 36,3 & 41,1 & 43,7 & 48,3 & 37,5 & 43,3 & 12,3 \\
\hline $\mathrm{GC}(\%)$ & 19,3 & 4,8 & 12,7 & 14,9 & 19,9 & 23,0 & 30,3 & 15,2 & 21,9 & 24,7 \\
\hline
\end{tabular}

Nota: PC - Peso Corporal; PM - perímetro Muslo; PG - perímetro gemelo; PBC - Perímetro Brazo Contraído; DH - Diámetro Humero; DF - Diámetro Fémur; LB - longitud del brazo; $\sum 7 \mathrm{PC}$ - sumatoria de los pliegues; PVC - Pico de la velocidad de crecimiento; MG - Masa Grasa; MLG - Masa Libre de Grasa; GC - porcentaje de grasa corporal.
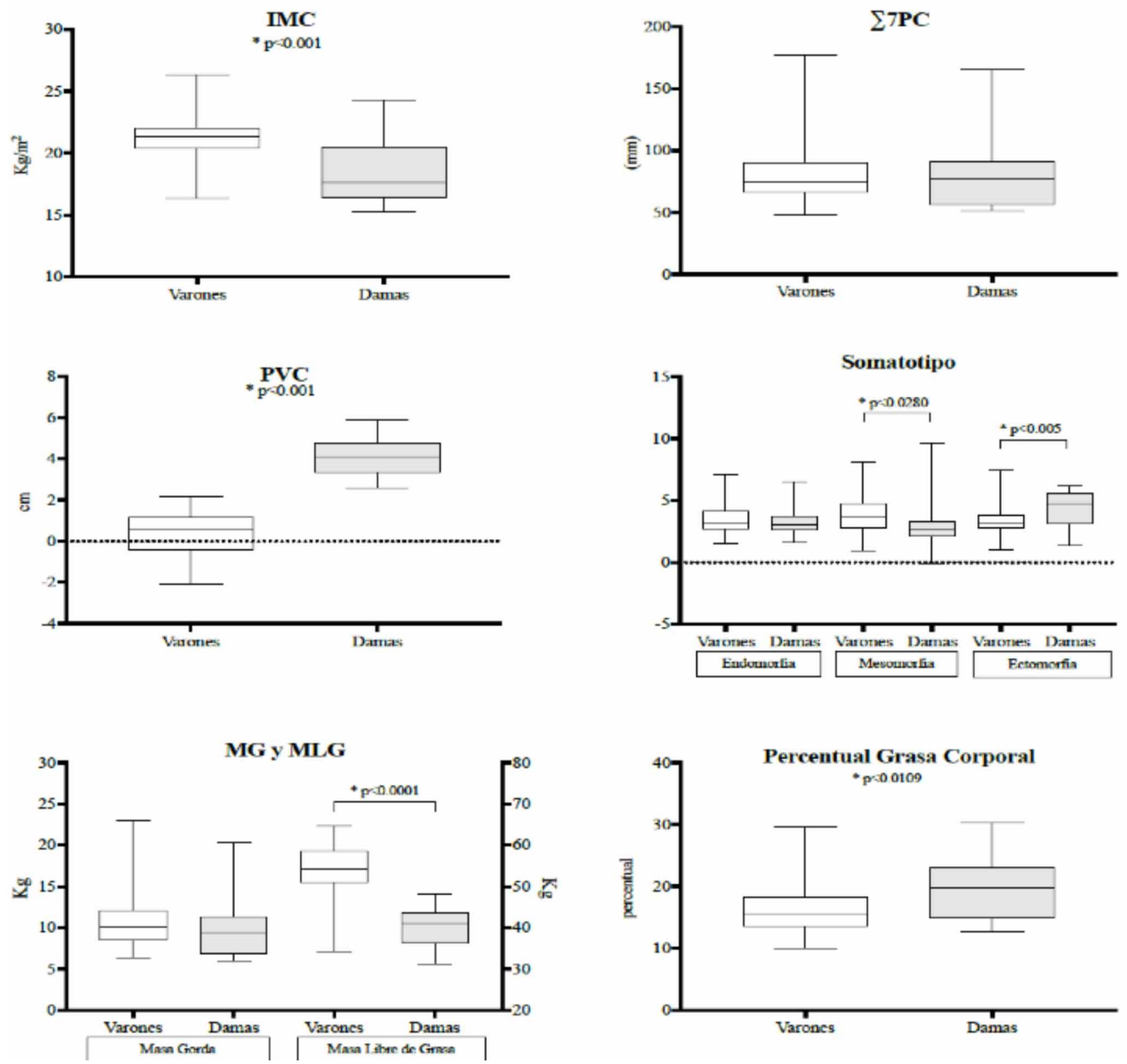

Fig 1. Comparación entre las medidas antropométricas y de Composición Corporal entre varones y damas tenistas de elite de 14-16 años. 
VILlOUTA, P. L.; CORREIA DE CAMPOS, L. F. C.; PAREDES, A. M.; VARGAS, V. R.; MARTíNEZ, S, C. \& ARANEDA, G. N. Caracterización antropométrica y composición corporal de tenistas de elite varones y damas de Chile. Int. J. Morphol., 39(1):84-89, 2021

\section{DISCUSIÓN}

En relación a los resultados obtenidos, los jugadores de tenis varones mostraron un valor promedio de peso corporal de $64,3 \pm 7,94 \mathrm{~kg}$, valores similares a los reportados en tenistas juveniles, por Sánchez-Muñoz et al. $69,9 \pm 6,8 \mathrm{~kg}$, Pereira, 63,78 \pm 7,6 kg, Cócaro et al. 63,80 \pm 6,86 kg, Torres Luque et al. 66,13 $\pm 10,58 \mathrm{~kg}$, Ziemann et al. $66.0 \pm 14,8 \mathrm{~kg}$. Por su parte, se observaron valores menores de Peso Corporal

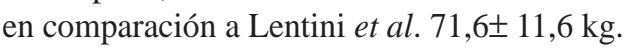

Por parte de las tenistas damas, se obtuvo una media de peso corporal 49,8 $\pm 6,9 \mathrm{~kg}$, consistente con los hallazgos de Ziemann et al. $52.8 \pm 6.8 \mathrm{~kg}$, por su parte son diferentes a los reportados por Lentini et al. 60,9 8,8 kg, Sánchez-Muñoz et al. 59,9 $\pm 6,2 \mathrm{~kg}$, Pereira, 59,1 $\pm 9,05 \mathrm{~kg}$, Torres Luque $e t$ $a l .54,18 \pm 7,70 \mathrm{~kg}$, que encontraron mayores valores de peso corporal.

En correspondencia, al porcentaje grasa, los valores obtenidos para los varones de $16,6 \pm 4,1 \%$, son similares a los resultados de Sánchez-Muñoz et al. 15,2 $\pm 2,4 \%$, Cócaro et al. 18,3 $\pm 5,9 \%$, Torres Luque et al. 12,67 $\pm 2,56 \%$, En cambio, mostraron mayor porcentaje de grasa que los valores obtenidos por Pereira, 10,6 $\pm 2,88 \%$, Ziemann et al. 9,7 $\pm 3,1 \%$.

En cuanto al porcentaje de grasa de las damas, la media fue $19,3 \pm 4,8 \%$, lo que es similar a los datos de Ziemann et al. 18,3 $\pm 2,8 \%$, Pereira, 20,2 $\pm 5,1 \%$, Torres Luque et al. $16,27 \pm 4,26 \%$, y con valores más bajos que los de SánchezMuñoz et al.28.5 $\pm 3.7 \%$.

En atención a los resultados con respecto al porcentaje de grasa podemos decir que, este indicador está relacionado al rendimiento deportivo una vez que la masa grasa puede disminuir parámetros de potencia, velocidad y agilidad en los jugadores de tenis. (Kovacs et al., 2007; Pradas de la Fuente et al.).

Para la estatura, los tenistas varones muestran un promedio fue de $1,74 \pm 0,08 \mathrm{~m}$, similar a lo encontrado por Sánchez-Muñoz et al. 1,76 $\pm 0,06 \mathrm{~m}$, Cócaro et al. 1,75 $\pm 0,07$ $\mathrm{m}$, Pereira, 1,76 $\pm 0,05 \mathrm{~m}$, Torres Luque et al. $174 \pm 0,06 \mathrm{~m}$, Lentini et al. 1,77 $\pm 0,08 \mathrm{~m}$; Ziemann et al.1,76 $\pm 0,10$.

Para la estatura de las damas, el promedio fue de 1,65 \pm 0,03 m, similar a los resultados de Sánchez-Muñoz et al. 1,65 $\pm 0,06 \mathrm{~m}$, Lentini et al. 1,60,06 m, Pereira, 1,67 $\pm 0,07 \mathrm{~m}$, Torres Luque et al. 1,62 \pm 0,06 m, Ziemann et al. 1,64 $\pm 0,06 \mathrm{~m}$.

Respecto al rendimiento deportivo, la estatura es un elemento necesario de considerar en cualquier modalidad deportiva, es así como en varias ocasiones se intenta explicar que la capacidad de los tenistas de moverse ágilmente por la cancha se debe a la talla, el peso o la composición corporal (Ziemann et al.)

Con relación a los valores obtenidos para el somatotipo, en el caso de los varones, el componente Mesomorfo balanceado obtenido, es similar a los resultados de Sánchez-Muñoz et al., Torres Luque et al., Lentini et al. Por su parte, Pereira, encontró un predominio del componente Ectomesomorficoen sus tenistas evaluados. En este sentido, un mayor componente muscular ha sido relacionado con el nivel de entrenamiento, la capacidad física de los deportistas y con los requerimientos motores del tenis, caracterizado por movimientos explosivos y veloces. (Ziemann et al.; Bompa \& Buzzichelli, 2015).

En cuestión para las damas, el somatotipo, presentó una tipología Ectomorfo balanceada, lo que no coincide con lo reportado por Sánchez-Muñoz et al. Mesoendomórfico; Lentini et al. Mesoectomorfico; Pereira, Endoectomórfico; y Endomesomorfico en Torres Luque et al. Lo anterior, parece ser relevante ya que indicaría que las jóvenes chilenas evaluadas, presentan características corporales que afectan su rendimiento deportivo, en especial, el desarrollo de golpes y movimientos veloces y potentes, necesarios para el éxito en esta modalidad deportiva (Pardos-Mainer et al., 2017).

Al comparar entre los sexos, se evidencia una mayor masa muscular en los varones y una menor concentración de grasa corporal con respecto a las Damas, lo que coincide con lo encontrado por Sánchez-Muñoz et al., Torres Luque et al., Lentini et al. Esta diferencia es atribuida al desarrollo biológico de los jugadores masculinos y femeninos en la etapa de la adolescencia, aspecto ya destacado en jugadores de tenis (Tuda et al., 1996; Lentini et al.).

En concordancia al análisis del crecimiento físico por edad biológica (PVC), se observó que tanto los varones como las damas están de acuerdo a lo esperado para su maduración biológica ( $\mathrm{V}=0,4 \pm 0,9 ; \mathrm{D}=4,1 \pm 0,9)$ (Martin et al., 2004; Malina et al., 2007).

Este estudio presenta como limitaciones, la selección no-probabilística y cantidad de muestra evaluada, lo que limita sus generalizaciones a otras poblaciones, sumado a la falta de control de la interacción con otras cualidades físicas y motoras determinantes en el rendimiento deportivo, lo que podría enriquecer futuros estudios en la temática. Por su parte, las principales fortalezas del estudio, es que se aborda una temática poco explorada a nivel nacional y sudamericano, y además aplica procedimientos y pruebas fiables, sencillas y económicas, pudiendo ser replicadas con facilidad. 
En conclusión podemos describir que, los resultados de ambos grupos muestran características antropométricas y de composición corporal similares a otros grupos de tenistas juveniles, a excepción del somatotipo en el caso de las damas. El estudio, a su vez, evidencia diferencias entre varones y damas para el porcentaje de grasa corporal, masa libre de grasa, PVC y somatotipo.

Estos resultados obtenidos pueden servir de referencia para la planificación del entrenamiento, la competencia y la selección de jóvenes tenistas chilenos, además se trata de una publicación inédita para la modalidad deportiva en Chile, por lo que consideramos necesario continuar y profundizar el incipiente conocimiento científico asociado a la formación deportiva inicial.

VILLOUTA, P. L.; CORREIA DE CAMPOS, L. F. C.; PAREDES, A. M.; VARGAS, V. R.; MARTÍNEZ, S, C. \& ARANEDA, G. N. Anthropometric characterization and body composition of male and female elite tennis in Chile. Int. J. Morphol., 39(1):84-89, 2021.

SUMMARY: The aim of the study was to determine the anthropometric characteristics and body composition in young Chilean elite tennis players aged 14 to 16 years. It was a descriptive crosssectional study. A non-probability convenience sampling of 84 tennis players (58 males $15.5 \pm 0.76$ years old and 26 females, $15.3 \pm 0.8$ years old) was selected. Anthropometric variables of weight, height, sitting height, skin folds (biceps, triceps, subscapular, supraspinal, abdominal, anterior thigh and medial leg), perimeters (flexed arm, middle thigh and leg) and diameters (humerus and femur) were evaluated. Anthropometric analysis and calculations were performed to determine the body composition and somatotype of the participanting subjects. The results for males were, body weight $64.3 \pm 7.94 \mathrm{~kg}$, height $1.74 \pm$ $0.08 \mathrm{~m}$, percentage of fat $16.6 \pm 4.1 \%$, balanced mesomorph component. The females had a body weight of $49.8 \pm 6.9 \mathrm{~kg}$, height of $1.65 \pm 0.03$ $\mathrm{m}$, percentage of fat $19.3 \pm 4.8 \%$, balanced ectomorph component. In general, both groups showed similar anthropometric and body composition characteristics as compared to other groups of young tennis players, with the exception of the somatotype in the case of the females.

KEY WORDS: Tennis; Anthropometrics; Body composition; Somatotype.

\section{REFERENCIAS BIBLIOGRÁFICAS}

Bompa, T. O. \& Buzzichelli, C. A. Periodización del Entrenamiento Deportivo. $4^{\mathrm{a}}$ ed. Barcelona, Paidotribo, 2015.

Carter, L. \& Heath, B. Somatotyping, Development and Applications. Cambridge, Cambridge University Press, 1990.

Cócaro, E. S.; Priore, S. E.; da Costa, R. F. \& Fisberg, M. Food intake and anthropometric profile of adolescent tennis players. Nutrire Rev. Soc. Bras. Aliment. Nutr., 37(3):293-308, 2012.

Cossio-Bolaños, M.; Vidal-Espinoza, R.; Lagos- Luciano, J. \& Gómez-Campos, R. Perfil antropométrico en función del estado nutricional de niños con discapacidad intelectual. Rev. Chil. Pediatr., 86(1):18-24, 2015.

Guedes, D. P. \& Rechenchosky, L. Comparação da gordura corporal predita por métodos antropométricos: índice de massa corporal e espessuras de dobras cutâneas. Rev. Bras. Cineantropom. Desempenho Hum., 10(1):1-7, 2008.
Kovacs, M. S.; Pritchett, R.; Wickwire, P. J.; Green, J. M. \& Bishop, P. Physical performance changes after unsupervised training during the autumn/spring semester break in competitive tennis players. Br. J. Sports Med., 41(11):70510, 2007.

Kramer, T.; Huijgen, B. C. H.; Elferink-Gemser, M. T. \& Visscher, C. A longitudinal study of physical fitness in elite junior tennis players. Pediatr. Exerc. Sci., 28(4):553-64, 2016.

Lentini, N. A.; Gris, G. M.; Cardey, M. L.; Aquilino, G. \& Dolce, P. A. Estudio somatotípico en deportistas de alto rendimiento de Argentina. Arch. Med. Deporte, 21(104):497-509, 2004.

Malina, R. M.; Bouchard, C. \& Bar-Or, O. Growth, Maturation and Physical Activity. $2^{\mathrm{a}}$ ed. Champaign (IL), Human Kinetics, 2004.

Malina, R. M.; Ribeiro, B.; Aroso, J. \& Cumming, S. P. Characteristics of youth soccer players aged 13-15 years classified by skill level. Br. J. Sports Med. 41(5):290-5, 2007

Martin, D.; Nicolaus, J.; Ostrowski, C. \& Rost, K. Metodología General del Entrenamiento Infantil y Juvenil. Barcelona, Paidotribo, 2004.

Mirwald, R. L.; Baxter-Jones, A. D. G.; Bailey, D. A. \& Beunen, G. P. An assessment of maturity from anthropometric measurements. Med.Sci. Sports Exerc., 34(4):689-94, 2002.

Pardos-Mainer, E.; Ustero-Pérez, O. \& Gonzalo-Skok, O. Efectos de un entrenamiento pliométrico en extremidades superiores e inferiores en el rendimiento físico en jóvenes tenistas. RICYDE Rev. Int. Cienc. Deporte, 13(49):225-43, 2017.

Peña, J.; Moreno-Doutres, D.; Coma, J.; Cook, M. \& Buscà, B. Anthropometric and fitness profile of high-level basketball, handball and volleyball players. Rev. Andal. Med. Deporte, 11(1):30-5, 2018.

Pereira, C. Perfil corporal de tenistas participantes do campeonato brasileiro de tênis, ambos os sexos, categoria 16 anos: um relato cineantropométrico. Rev. Treinamento Desport., 6(1):53-71, 2001.

Pradas de la Fuente, F.; Carrasco Páez, L.; Martínez Pardo, E. \& Herrero Pagán, R. Perfil antropométrico, somatotipo y composición corporal de jóvenes jugadores de tenis de mesa. Rev. Int. Cienc. Deporte, 3(7):11-23, 2007.

Ross, W. D. \& Marfell-Jones, M. J. Kinanthropometry. In: MacDougall, J. D.; Wenger, H. A. \& Geen, H. J. (Eds.). Physiological Testing of Elite Athlete. London, Human Kinetics, 1991.

Sánchez-Muñoz, C., Sanz, D. \& Zabala, M. Anthropometric characteristics, body composition and somatotype of elite junior tennis players. Br. J. Sports Med., 41(11):793-9, 2007

Slaughter, M. H.; Lohman, T. G.; Boileau, R. A.; Horswill, C. A.; Stillman, R. J.; Van Loan, M. D. \& Bemben, D. A. Skinfold equations for estimation of body fatness in children and youth. Hum. Biol., 60(5):709-23, 1988.

Torres Luque, G.; Alacid Cárceles, F.; Ferragut Fiol, C. \&Villaverde Gutiérrez, C. Estudio cineantropométrico del jugador de tenis adolescente. Cult. Cienc. Deporte, 2(4):27-32, 2006.

Tuda, M.; Rodríguez Guisado, F. A. \& Solanellas Donato, F. Valoración cineantropométrica de tenistas de diferentes categorías. Apunts Educ. Fís. Deportes, (44-45):122-35, 1996.

Zemel, B. S.; Riley, E. M. \& Stallings, V. A. Evaluation of methodology for nutritional assessment in children: anthropometry, body composition, and energy expenditure. Ann. Rev. Nutr., 17:211-35, 1997.

Ziemann, E.; Sledziewska, E.; Grzywacz, T.; Gibson, A. L. \& Wierzba, T. H. Body composition and physical capacity of elite adolescent female tennis players. Georgian Med. News, (196-197):19-27, 2011.

\section{Dirección para correspondencia: \\ Rodrigo Vargas Vitoria}

Facultad de Ciencias de la Educación

Universidad Católica del Maule

Doctorado en Ciencias de la Actividad Física UCM

Talca

CHILE

Email: rvargas@ucm.cl

Recibido: 03-02-2020

Aceptado: 25-09-2020 